ORIGINAL ARTICLE

\title{
Impact of adverse publicity on MMR vaccine uptake: a population based analysis of vaccine uptake records for one million children, born 1987-2004
}

\author{
V Friederichs, J C Cameron, C Robertson
}

Arch Dis Child 2006;91:465-468. doi: 10.1136/adc.2005.085944

See end of article for authors' affiliations

......................

Correspondence to:

Dr J C Cameron, Health

Protection Scotland, Clifton

House, Clifton Place,

Glasgow G3 7LN, UK;

claire.cameron@hps.scot.

nhs.uk

Accepted 1 March 2006

\begin{abstract}
Aims: To determine the impact of adverse publicity on MMR uptake and measles susceptibility, including whether vaccination is delayed and the role of deprivation.

Methods: A population database for all Scotland containing immunisation records for over one million children ( $n=1079$ 327) born 1987-2004 was analysed. MMR uptake was determined by birth cohort and deprivation category. "Final" uptake (at approx age 6 years) was predicted by linear regression by birth cohort. Measles susceptibility in 1998 and 2003 was determined by postcode sector and district for cohorts combined to construct nursery and primary school age groups.

Results: There is evidence of a slight rise in late uptake, but insufficient to compensate for underlying declines. Late vaccination continues to be associated with deprivation, while the most affluent tend to be vaccinated promptly, or not at all. Predicted figures for "final" MMR1 uptake are over $90 \%$, but under $95 \%$. Measles susceptibility has increased significantly in nursery children, with an eightfold rise in the number of districts with greater than $20 \%$ susceptibility in this group (from 3 to 25).

Conclusions: Increased measles susceptibility in nursery children is concerning, particularly in the most vulnerable areas. These figures are likely to increase in the future, as MMR uptake has not yet returned to the previous higher level. Increased susceptibility levels can also be expected in primary schools in the future, as levels of late uptake are insufficient to compensate. Predicted figures for "final" MMR1 uptake are under the herd immunity threshold and campaigns may be required to increase uptake among future primary school children.
\end{abstract}

$M$ MR vaccine is the safest and most effective way to protect against measles, mumps, and rubella. It is recommended in the UK at ages 13 months (MMRI) and 3 years 4 months to 5 years (MMR2), ${ }^{1}$ although uptake has decreased following prominent adverse publicity since 1998. ${ }^{23}$ We analysed MMR uptake across Scotland from its introduction (October 1988) to most recent data (February 2005). This would enable any changes in uptake to be detected, including whether parents are delaying vaccination, and whether there are differences by deprivation, and allow prediction of final MMRl uptake. These figures would also allow measles susceptibility among nursery and primary school children to be examined, including in small geographical areas, where clustering of non-immunes could be detected.

\section{METHODS}

Data on vaccines received by children throughout Scotland are held on the Scottish Immunisation and Recall System (SIRS). On 15 February 2005, this contained over one million entries $(\mathrm{n}=1079327)$ for children born between 1 January 1987 and 31 December 2004. Records include date of MMR vaccination, date of birth, sex, postcode sector of residence (e.g. G3 7--), and deprivation category ( 1 to 7 ). Deprivation is determined according to the proportion of the population in the postcode sector with access to a car, in overcrowded households, with the head of household in social class IV or $\mathrm{V}$, and in households with unemployed men, using the Carstairs and Morris index. ${ }^{4}$ All children are entered onto the database, at birth or on migration. Measures in place to ensure a high degree of data completeness and accuracy include unique personal identifiers, incentive payments, and data checking by NHS Boards. However, the data are minimum figures for vaccine uptake, as under-recording of data is possible, ${ }^{5}$ and data on single vaccines cannot be entered.

To investigate delayed vaccination and any impact of deprivation, MMRl uptake, after age 2 years, was examined by deprivation category. MMRl uptake tends to stabilise for birth cohorts at around age 6 years. Figures for this "final" uptake by birth cohort were predicted by linear regression, based on the most recent data.

To estimate measles susceptibility, we assumed a vaccine efficacy of $90 \%$ for one dose and $99 \%$ for two doses. ${ }^{6}$ The percentage of susceptible nursery and primary school age children was estimated for individual postcode sectors, as at 1 September 1998 or 1 September 2003 (that is, approximately the beginning of the academic year). The primary cohort included children born between 1 March 1997 and 28 February 1999 (2003) or 1 March 1992 and 28 February 1994 (1998), as these dates are specified in school entry criteria. These children would have been aged 4.5 years to 6.5 years, be expected to be in primary years 1 and 2, and virtually all to have been offered MMRl and MMR2. The nursery cohort included children born between 1 March 1999 and 28 February 2001 (2003) or 1 March 1994 and 28 February 1996 (1998). These children would have been aged 2.5 to 4.5 years and virtually all in pre-school settings. All in this cohort will have been offered MMR1, and some MMR2.

All statistical calculations were performed using R. ${ }^{7}$ Ethical approval was not required.

\section{RESULTS}

The percentage of children who received MMRI at ages 2, 3, or after 4 years is shown in table 1 . The 1999 birth cohort was the first with decreased MMRl uptake, despite the 1997 birth 


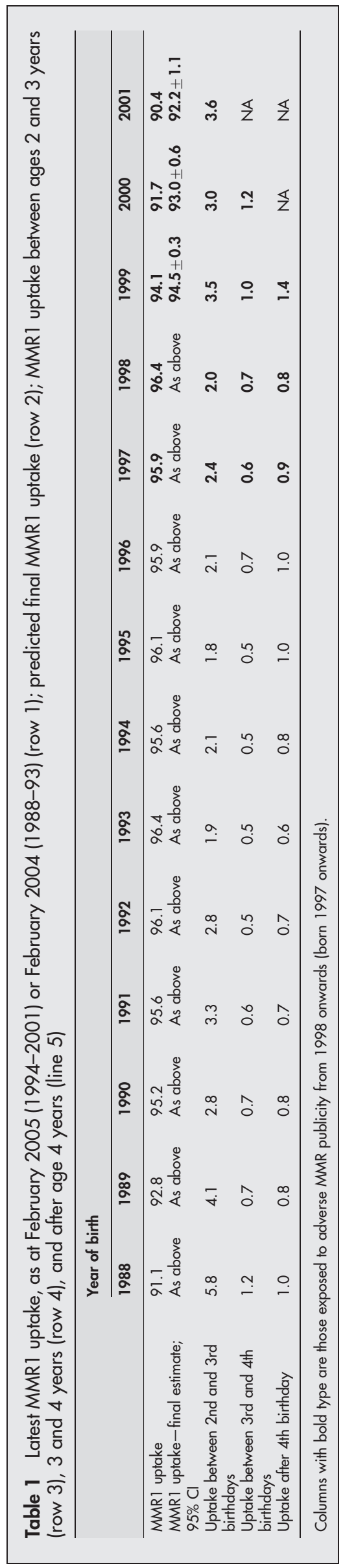

cohort being the first to be exposed to adverse publicity. There is also evidence of a slight increase in late MMRl uptake for the 1999 birth cohort onwards. The same overall pattern remains when data are analysed by deprivation (fig 1). However, the greater the deprivation, the greater the tendency for late vaccination, especially for the most deprived (category 7). Those who are more affluent tend to be vaccinated early, or not at all. The predicted "final" MMRl uptakes for birth cohorts 1999-2001 were all under 95\%, but over $90 \%$, including $95 \%$ confidence intervals. This is in contrast to birth cohorts 1992-98, which all have "final" MMRl uptake of around $96 \%$.

The percentage of primary children susceptible to measles did not change significantly from 1998 to 2003 and areas with greater than $10 \%$ susceptibility tended to be rural, with fewer children (table 2). Susceptibility among nursery children is higher as most have had access to MMRl only. The median susceptibility in postcode sectors increased significantly over the five years (from 13\% to 16.4\%) and the percentage of postcode sectors with greater than $20 \%$ susceptibility almost tripled (from $8.2 \%$ to $23.8 \%$ ). In 2003 , these included areas of higher population density (median 59 children), although areas still tended to have fewer children than those sectors with under $20 \%$ susceptibility (median 104 children). Looking at larger areas, of postcode district (e.g. G7 ---), and restricting analysis to districts with over 200 nursery children, in 1998, there were only three districts with greater than $20 \%$ susceptibility, but by 2003 , this figure had risen to 25, with an excess of deprivation categories 1 and 7 .

\section{DISCUSSION}

Late uptake of MMRl has increased slightly for birth cohorts 1999 onwards, but the increase is insufficient to compensate for general declines, which have led to significantly increased measles susceptibility in nursery children.

The study uses the entire immunisation dataset for Scotland and should therefore provide a complete picture of MMR uptake and measles susceptibility. It should be applicable elsewhere in the UK, according to deprivation, as the whole country experienced declines under similar media influences. It is interesting that despite the 1997 birth cohort being the first to be exposed to adverse publicity, it was only cohorts 1999 onwards that had significant uptake declines, suggesting a gradual build-up of its effect, rather than an instant impact.

Increases in measles susceptibility were predicted from decreases in MMR uptake, but this is the first study published to translate these into susceptibility estimates and examine effects, at the level of postcode districts and sectors. Although susceptibility among primary children is acceptably low in most areas, increased susceptibility among nursery children is concerning, particularly in the most vulnerable districts, where a significant outbreak could result should measles be introduced, creating further inequalities in areas of higher deprivation. Outbreaks have already been observed elsewhere in the UK, where uptake has been much lower and for a longer period. ${ }^{89}$

Factors influencing vaccine uptake have been widely investigated, with previous UK studies finding that MMRl uptake declined with increasing deprivation, ${ }^{10}$ and that living with one parent only ${ }^{11}{ }^{12}$ or being the third or more child was significantly associated with lower uptake, ${ }^{12}$ while unemployment was significantly associated with increased uptake. ${ }^{11}$ However, a more recent study found that parents declining MMR vaccine were more likely to belong to a higher socioeconomic group. ${ }^{5}$ Lower MMR uptake has also been shown for children registered with inducement or single handed practices. ${ }^{13}$ These studies are very informative, but all measured uptake for a range of ages between 19 and 24 


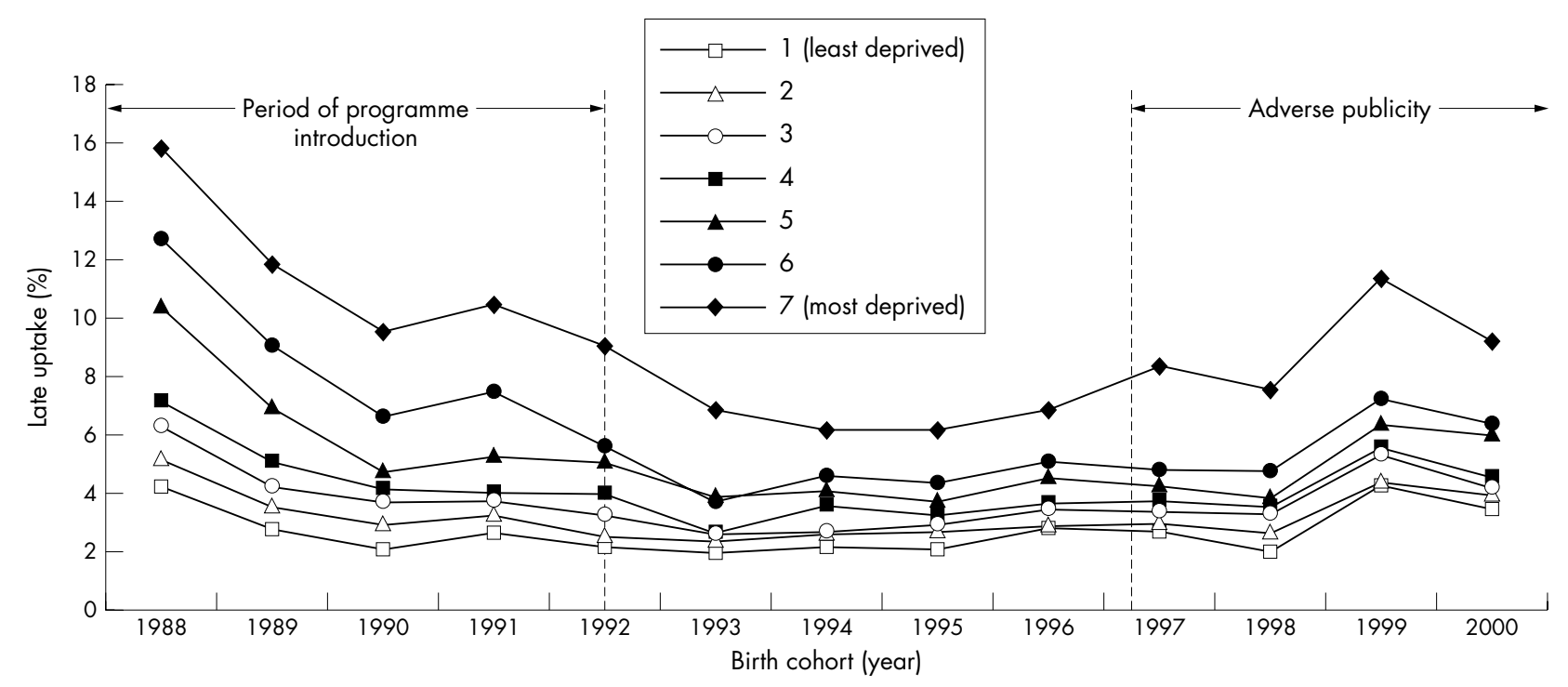

Figure 1 MMR uptake after age 2 years, by deprivation category, birth cohorts, 1988-2000.

Table 2 Measles susceptibility among nursery and primary age cohorts in Scotland, September 1998 and September 2003

\begin{tabular}{|c|c|c|c|c|}
\hline & \multicolumn{2}{|c|}{ Median susceptibility by postcode sector (IQR) } & \multicolumn{2}{|c|}{$\begin{array}{l}\text { Sectors with }>10 \% \text { or }>20 \% \text { susceptibility }(\%) \\
\text { [Median no. of children (IQR)] }\end{array}$} \\
\hline & 1998 & 2003 & $\overline{1998}$ & 2003 \\
\hline $\begin{array}{l}\text { Sectors with }>10 \% \text { susceptibility } \\
\text { Primary children }\end{array}$ & $4.8(2.7-7.1)$ & $4.4(2.9-6.7)$ & $11.5 \%[43(15-100)]$ & $10.5 \%[25(7-61)]$ \\
\hline $\begin{array}{l}\text { Sectors with }>20 \% \text { susceptibility } \\
\text { Nursery children }\end{array}$ & $13(10.9-15.2)$ & $16.4(13.3-19.8)$ & $8.2 \%[15(4-54)]$ & $23.8 \%$ [59 (12-114) \\
\hline
\end{tabular}

$I Q R$, interquartile range.

months, and did not examine delayed uptake after the age of 24 months, as presented here. The final figures predicted by linear regression are also new.

It is clear that improvements in MMR uptake are desirable. Any patterns associated with deprivation are important in understanding how to maximise uptake within a given social setting. It is most important to increase overall uptake in more deprived areas, but increased late uptake in more affluent areas should also be promoted. We have shown that those who are more affluent tend to make timely decisions as to whether to vaccinate or not, while those who are more deprived experience greater delay. We were unable to distinguish between individual (for example, educational attainment) and area level (for example, health service accessibility) factors that may determine this. Therefore, we are now investigating the association of more specific factors, such as car ownership, unemployment, overcrowding, social class, educational attainment, country of birth, and rurality with measles susceptibility, in a further study. This may clarify whether delays in vaccination are related to a longer process of decision making, or to more non-specific aspects such as accessibility, mobility, or perceptions of the health service. If it is the former, qualitative studies have shown that contact with health professionals and recommended health education material positively contribute to decision making. ${ }^{5}{ }^{14}$ There should therefore perhaps be greater awareness of this potential when working among more deprived communities. The potential for current and future outbreaks of measles, mumps, and rubella is currently being investigated in Scotland by techniques of mathematical modelling. These will further inform possible future interventions, such as initiatives to increase MMR uptake prior to school entry, which is likely to be required to increase MMRl uptake above $95 \%$, the herd immunity threshold.

\section{What is already known on this topic}

- There has been sustained adverse publicity for MMR vaccine since 1998

- MMR uptake has fallen, but the extent of delayed vaccination, the role of deprivation, predicted final figures, and the impact on measles susceptibility in small areas has not been published

\section{What this study adds}

- Late uptake has increased slightly and more affluent parents tend to vaccinate early or not at all, while the most deprived are more likely to experience delay

- Measles susceptibility has increased significantly in nursery children since 1998, with 25 postcode districts in 2003 having $>20 \%$ susceptibility. Final MMR1 uptake predictions for primary school children are $>90 \%$ but $<95 \%$, indicating a need for further immunisation to prevent future measles transmission 


\section{ACKNOWLEDGEMENTS}

We are grateful to the Scottish Executive for funding this project. We would also like to thank all who contribute to the collection of SIRS data, in particular the Immunisation Co-ordinators, the SIRS User Group (chaired by Dr David Cromie), and the Information and Statistics Division (Dr Jim Chalmers and Elaine Strange), and Dr Martin Donaghy, Health Protection Scotland for useful discussions.

\section{Authors' affiliations}

J C Cameron, Health Protection Scotland, Glasgow, Scotland, UK V Friederichs, C Robertson, Department of Statistics and Modelling Science, University of Strathclyde, Glasgow, Scotland, UK

Competing interests: Health Protection Scotland has received funding for research and conference attendance from pharmaceutical companies

\section{REFERENCES}

1 Department of Health, Welsh Office, Scottish Office Department of Health, DHSS (Northern Ireland). Immunisation against infectious disease. London: HMSO, 1996.

2 Cameron JC, Littler H. Vaccine-preventable and childhood disease. HPS Weekly Report 2005;39:286-7.

3 HPA. COVER programme: April to June 2005. Commun Dis Rep CDR Wkly [serial online] 2005;15(38), Immunisation.
4 Mcloone P. Carstairs scores for Scottish postcode sectors from the 1991 census. Glasgow: Public Health Resources Unit, 2000.

5 Macdonald H, Henderson R, Oates K. Low uptake of immunisation: contributing factors. Community Practitioner 2004;77(3):95-100.

6 Strebel PM, Papania MJ, Halsey NA. Measles vaccine. In: Plotkin SA, Orenstein WA, eds. Vaccines, 4th edn. Philadelphia: Saunders, 2004.

7 R Development Core Team. R: A language and environment for statistical computing. Vienna: R Foundation for Statistical Computing, 2005.

8 Ramsay ME, Jin L, White J, et al. The elimination of indigenous measles transmission in England and Wales. J Infect Dis 2003;187/suppl 1):S198-207.

9 Morgan OWC, Meltzer M, Muir D, et al. Specialist vaccination advice and pockets of resistance to MMR vaccination: lessons from an outbreak of measles. Commun Dis Public Health 2003;6:330-3.

10 Reading R, Colver A, Openshaw S, et al. Do interventions that improve immunisation uptake also reduce social inequalities in uptake? BM 1994:308:1142-4.

11 Sharland $M$, Atkinson $P$, Maguire $H$, et al. Lone parent families are an independent risk factor for lower rates of childhood immunisation in London. Commun Dis Rep Rev 1997;7(RR-11):R169-72.

12 Li J, Taylor B. Factors affecting uptake of measles, mumps, and rubella immunisation. BMJ 1993;307:168-71.

13 Henderson R, Oates K, Macdonald H, et al. Factors influencing the uptake of childhood immunisation in rural areas. Br J Gen Pract 2004:54:114-18.

14 Martin C, Sansom A. Evaluation of the MMR Discussion Pack and Preparation of MMR Communication Strategy. Edinburgh: NHS Health Scotland, 2003 (http://www.healthscotland.com/immunisation/mmr/ reports.cfm).

\section{Inhaled hypertonic saline in cystic fibrosis}

n the lungs of patients with cystic fibrosis there is excessive absorption of sodium and defective secretion of chloride across the respiratory epithelium. This leads to extra water absorption, drying of airway contents, and inspissation of mucus, with defective mucociliary function and consequent liability to infection. Now researchers in Australia (Mark R Elkins and colleagues. New England Journal of Medicine 2006;354:229-40; see also editorial, ibid: 291-3) and the USA (Scott H Donaldson and colleagues. Ibid: 241-50) have reported improvements in patients with cystic fibrosis after regular inhalation of hypertonic saline.

In Australia 164 patients aged over 5 years (mean 18 years) were treated for 48 weeks by random allocation to twice daily inhalations of hypertonic (7\%) saline or $0.9 \%$ saline. Standard treatments were continued and a bronchodilator was taken before each dose. Values for FVC and $\mathrm{FEV}_{1}$ increased significantly in the hypertonic saline group and they had fewer clinical exacerbations. At the end of the trial $76 \%$ of patients in the hypertonic saline group and $62 \%$ in the control group had been free of exacerbations.

In the American trial 24 patients aged over13 years (mean 26 years) inhaled nebulised hypertonic saline ( $5 \mathrm{ml}$ of $7 \%$ saline) four times daily for 2 weeks. Randomisation was to pretreatment with amiloride, a sodium-channel blocker, or placebo on the hypothesis that amiloride might prolong the duration of action of hypertonic saline. In the event, hypertonic saline improved mucus clearance and lung function, but only in the group that did not receive amiloride. In vitro studies showed that hypertonic saline induced a much larger increase in airway surface liquid in airway epithelium from people with cystic fibrosis than in control airway epithelium and that this was related to the function of the cystic fibrosis transmembrane regulator (CFTR) chloride channel.

Inhaled hypertonic saline may be a beneficial add-on treatment for patients with cystic fibrosis. It is time consuming and there are doubts about whether patients will tolerate it. The editorialist mentions dry powder, metered-dose inhalations of mannitol, currently under trial and potentially an alternative osmotic agent. 